\title{
p38 MAPK Contributes to the Growth Inhibition of Leukemic Tumor Cells Mediated by Human Umbilical Cord Mesenchymal Stem Cells
}

\author{
Kun Tian, Shaoguang Yang, Qian Ren, Zhibo Han, Shihong Lu, \\ Fengxia Ma, Lei Zhang and Zhongchao Han
}

State Key Laboratory of Experimental Hematology, Institute of Hematology and Blood Disease Hospital, Chinese Academy of Medical Sciences and Peking Union Medical College, Tianjin

\section{Key Words}

Mesenchymal stromal cells • Leukemic tumor cells • Growth inhibition - Cell cycle arrest - p38 MAPK • SB203580 • SiRNA

\begin{abstract}
Background/Aims: Mesenchymal stem cells (MSCs) have been implicated in antitumor therapy for hematopoietic and non-hematopoietic tumors. Cellcontact and soluble factors are demonstrated to play a role in the growth inhibition of tumor cells mediated by MSCs in vitro, while there is little clue about signaling pathways involved in the process. P38 MAPK has been implicated as a suppressor of cell proliferation and tumorigenesis. We here investigate whether p38 MAPK is involved in MSC-induced growth inhibition of leukemic tumor cells. Methods: We characterized the effect of human umbilical cord mesenchymal stem cells (UC-MSCs) on proliferation, cell cycle and phosphorylation pattern of p38 MAPK in HL60 and K562 cells. SB203580, a specific inhibitor of p38 MAPK, or p38 MAPK-small interfering RNA (siRNA), were used to identify the role of p38 in growth suppression by UC-MSCs. We also investigated the expression of cell cycle regulators. Results: Treatment with UC-MSCs led to potent proliferation-inhibition of
\end{abstract}

\section{KARGER}

Fax +4161306 1234

E-Mail karger@karger.ch www.karger.com
(C) 2010 S. Karger AG, Basel

$1015-8987 / 10 / 0266-0799 \$ 26.00 / 0$

Accessible online at: www.karger.com/cpb
HL60 and K562 cells without inducing apoptosis. Growth inhibition by UC-MSCs was due to G0/G1 arrest. UC-MSCs increased phosphorylation of p38 MAPK in HL60 and K562 cells. Pharmacological inhibition or genetic silencing (through siRNA) of p38 MAPK partially abrogated the proliferationsuppression and cell cycle arrest caused by UCMSCs. UC-MSCs also modulated the expression of cell cycle regulatory proteins in HL60 and K562 cells while SB203580 reversed the effect. Conclusion: Taken together, our findings indicate that p38 MAPK is critical for the growth inhibitory effect of UC-MSCs on leukemic tumor cells.

Copyright @ 2010 S. Karger AG, Basel

\section{Introduction}

Mesenchymal stem cells derived from bone marrow (BM-MSCs) exhibit a potent anti-proliferative effect on tumor cells of hematopoietic and non hematopoietic origin in vitro [1]. The growth inhibition was caused by the arrest of tumor cells in the G1 phase of the cell cycle,

Professor Dr. Zhongchao Han

State Key Laboratory of Experimental Hematology, Institute of Hematology Chinese Academy of Medical Sciences and Peking Union Medical College Tianjin, 288 Nan Jing Road 300020 (P. R. China)

Tel. +86-22-27210717, Fax +86-22-66211430, E-Mail tihzchan@public.tpt.tj.cn 
accompanied by decreased expression of cyclin D2. Recently, Khakoo et al. [2] showed that MSCs exert potent antitumorigenic effects in a model of kaposi's sarcoma. The in vivo tumor-suppressing effect of MSCs correlates with their ability to inhibit the AKT activity in target cells in a contact-dependent manner. Yet in vitro MSCs can inhibit the activation of the Akt protein kinase within some but not all tumor and primary cell lines. Zhu et al. [3] further showed that MSCs can inhibit tumorcell proliferation in a contact-independent manner and identified DKK-1 (dickkopf-1), secreted by MSCs and acting as a negative regulator of WNT signaling pathway, to be one of the molecules responsible for the inhibitory effect. However, the growth rate of tumor cells was not fully restored to the normal level by RNA interference of DKK-1 or by neutralizing antibodies against DKK-1. This indicates that other pathways may be involved in MSCmediated inhibition of tumor growth.

The mitogen-activated protein kinases (MAPKs) are members of intracellular kinases that serve as focal points for diverse extracellular stimuli and function to regulate various cellular processes. The p38 MAPK is known to be activated by various cytokines and stressful stimuli and thus plays an essential role in cellular responses including cell proliferation, differentiation and apoptosis [4]. For example, p38 MAPK is a key negative regulator of cell proliferation in several cell types, including cardiomyocytes [5], corneal epitheliums [6] and umbilical vascular endothelial cells [7]. One remarkable role for the p38 MAPK pathway is the regulatory effect on G1/S as well as G2/M cell cycle checkpoint in response to cellular stress [8]. Negative regulation of cycin D at both the transcription and post-transcriptional levels was proposed as a potential target for p38 MAPK in the regulation of G1/S progression. Besides, $\mathrm{p} 38 \mathrm{MAPK}$ has recently been revealed as a suppressor of tumor development [9]. Inactivation of this pathway supports cellular transformation in vitro and promotes cancer development in mouse cancer models [10]. Also, downregulatioin of p38 MAPK were reported to trigger proliferative responses in quiescent leukemia cells [11]. We therefore investigated whether activation of $\mathrm{p} 38$ MAPK is involved in the suppression of leukemia cells mediated by MSCs.

In this study, we examined the growth inhibitory effects of human umbilical cord mesenchymal stem cells (UC-MSCs) on leukemic tumor cells, and we further investigated whether $\mathrm{p} 38$ MAPK signaling pathway was involved in the process. We found that p38 MAPK contributed to the proliferation inhibition, cell cycle arrest and modulation of cell cycle regulators in leukemic tumor cells caused by UC-MSCs. These results suggest that p38 MAPK might be a potential therapeutic target for treatment of leukemic tumors.

\section{Materials and Methods}

\section{Reagents}

The antibodies against phospho-p38MAPK, p38MAPK, p2 $7^{\text {Kip1 }}$, and cyclin D2 were purchased from Cell Signaling Technology (Beverly, MD, USA), Actin was from Santa Cruz. SB203580 was from Sigma.

\section{Tumor cell culture}

The human leukemic tumor cell lines HL60 and K562 cells were maintained in RPMI 1640 medium (Gibco Life Technologies, Paisley, UK) supplemented with $10 \%$ fetal calf serum (FCS) in a humidified atmosphere with $5 \% \mathrm{CO}_{2}$ at $37^{\circ} \mathrm{C}$. Synchronized leukemic tumor cells, when indicated, was achieved by incubation in RPMI 1640 medium with $0.1 \%$ FCS for $24 \mathrm{~h}$.

\section{Isolation and culture of UC-MSCS}

UC-MSCs were isolated from umbilical cords obtained from local maternity hospitals with donors' informed consent. Human tissue collection for research was approved by the institutional review board of the Chinese Academy of Medical Science and Peking Union Medical College. The details of isolation, ex vivo expansion and identification of UC-MSCs were essentially as described previously [12]. Cells of passage four to six were used in this study. UC-MSCs (30 Gy irradiated) were preplated and allowed to adhere overnight at $37^{\circ} \mathrm{C}$ before co-culture with tumor cells.

\section{Proliferation assays}

Tumor cells $\left(10^{4}\right.$ per well) were seeded in flat-bottom 96well plates, and treated with indicated doses of UC-MSCs for $72 \mathrm{~h}$. The proliferation of tumor cells was measured using the cell proliferation BrdU ELISA kit (Roche Diagnostics, Mannheim, Germany) according to manufacturer's instructions. $\mathrm{BrdU}$ was added for the last $18 \mathrm{~h}$ of incubation. After fixation, the mouse monoclonal anti-BrdU-POD antibody was added. The immune complexes were revealed by the subsequent substrate reaction, and quantified by measuring the absorbance at $405 \mathrm{~nm}$ with a reference wavelength at $490 \mathrm{~nm}$ in a microliter plate reader.

\section{Cell cycle analysis}

Tumor cells were plated into 6-well culture plates at a density of $1 \times 10^{6} /$ well and were cultured with or without UCMSCs $\left(10^{5}\right.$ per well) for $48 \mathrm{~h}$ before harvested and quantified. $10^{6}$ tumor cells were fixed with $70 \%$ cold ethanol at $4^{\circ} \mathrm{C}$ for more than $12 \mathrm{~h}$, washed with phosphate-buffered saline twice. The cells were then incubated with $50 \mu \mathrm{g} / \mathrm{ml}$ RNase A (Sigma) for $20 \mathrm{~min}$ at $37^{\circ} \mathrm{C}$ and stained with $50 \mathrm{mg} / \mathrm{ml}$ propidium iodide

Tian/Yang/Ren/Han/Lu/Ma/Zhang/Han 
A

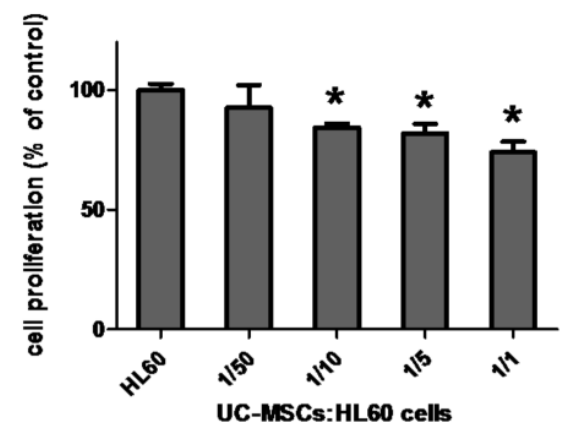

B

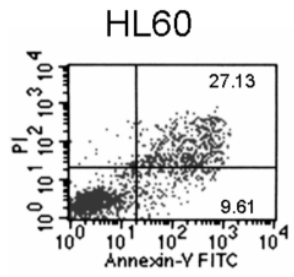

C

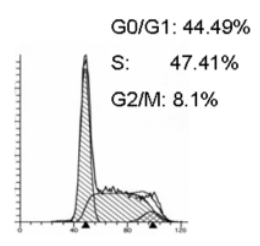

HL60
HL60+MSC
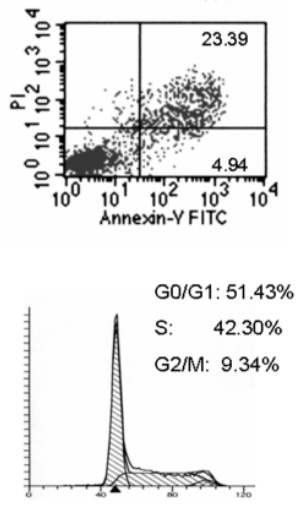

HL60+MSC
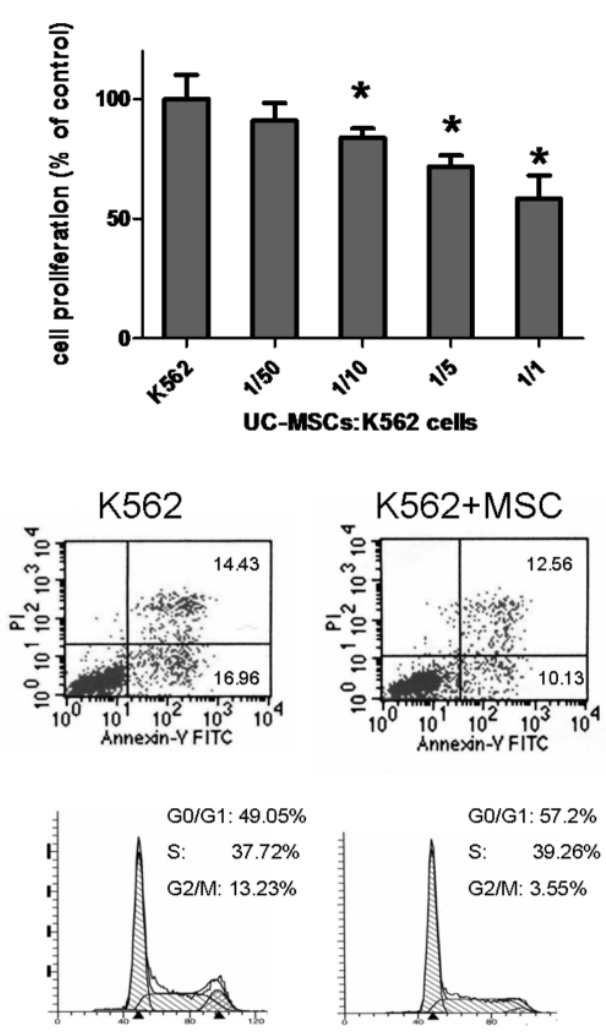

K562

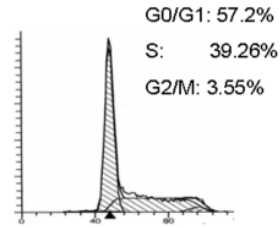

K562+MSC

Fig. 1. UC-MSCs inhibit proliferation of HL60 and K562 cells by G0/G1 arrest without induction of apoptosis. (A) Proliferation inhibitory effect of UC-MSCs on tumor cells. Tumor cells in 96 -well plates $\left(10^{4}\right.$ per well) were treated with grading doses of UCMSCs for $72 \mathrm{~h}$ and the cell proliferation was measured by BrdU ELISA kit. The ability of UC-MSCs to inhibit cell proliferation was determined by comparing with those cells without UC-MSCs treatment (\% of control) and each bar represents the mean \pm SD of triplicate determinations. The data are representative of three independent experiments. ${ }^{*} \mathrm{P}<0.05$ versus untreated cells. (B) UCMSCs protect tumor cells from apoptosis. Tumor cells in 6 -well plates $\left(10^{6}\right.$ per well) were maintained in RPMI 1640 with $1 \%$ FCS for $48 \mathrm{~h}$ with or without UC-MSCs $\left(10^{5}\right.$ per well) and cell apoptosis was evaluated by annexin V staining and PI incorporation. (C) UC-MSCs block HL60 and K562 cells at G0/G1 phase. Synchronized tumor cells $\left(10^{6}\right)$ cultured with or without UC-MSCs $\left(10^{5}\right)$ for $48 \mathrm{~h}$ were analyzed for cell-cycle progression as described in Materials and methods. Percentages of cells in each phase are shown in the upper corner of each cell-cycle graph. The results are representative of three independent experiments.

(PI) (Sigma) at room temperature for $5 \mathrm{~min}$. DNA content was analyzed in a FACScalibur flow cytometer (BD) using MODFIT software (Verity).

\section{Apoptosis analysis}

Tumor cells $\left(10^{6}\right.$ per well) were plated into 6-well culture plates and were cultured with $1 \%$ FCS in the presence or absence of UC-MSCs ( $10^{5}$ per well) for $48 \mathrm{~h}$. The AnnexinV/ PI apoptosis detection kit from BD was used for apoptosis assay according to manufacturer's instructions. Briefly, at least $10^{6}$ tumor cells were washed and resuspended in binding buffer (1:10 diluted in phosphate-buffered saline). Then the cells were incubated with AnnexinV $(5 \mu \mathrm{L})$ and PI $(5 \mu \mathrm{L})$ for $15 \mathrm{~min}$. For every condition, 15,000 events were collected and analyzed by flow cytometer.

\section{Small interfering RNA transfection}

Specific small interfering RNAs (siRNAs) targeting p38 MAPK (sip38, SignalSilence ${ }^{\circledR}$ p38 MAPK siRNA) and negative control siRNAs (siCtrl, SignalSilence ${ }^{\circledR}$ Control siRNA) were purchased from Cell Signaling Technology (Beverley, MD, USA). HL60 and K562 cells were transfected with doublestranded siRNA using the Hiperfect Transfection Reagent (Qiagen) according to the manufacturer's instructions. In brief, $6 \mu \mathrm{l}$ of Hiperfect Transfection Reagent and finally $100 \mathrm{nM}$ of siRNA were diluted with serum-free RPMI 1640 medium, preincubated at room temperature for $10 \mathrm{~min}$ and then added to the tumor cells maintained in RPMI 1640 medium containing $10 \%$ FCS. Tumor cells were incubated with transfection complexes at $37^{\circ} \mathrm{C}$ for $72 \mathrm{~h}$ and then the p38 MAPK protein level was detected by western blot to assess silence efficiency. 


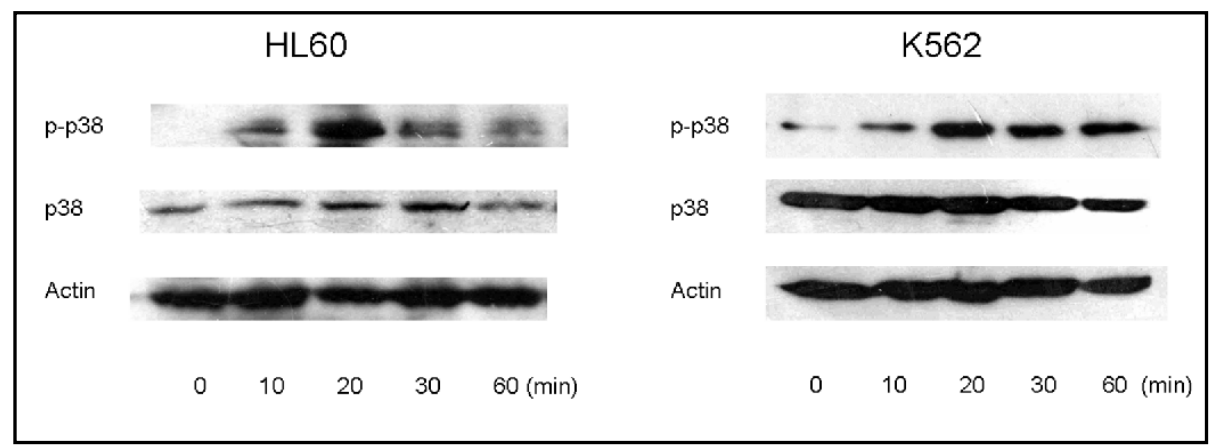

Fig. 2. UC-MSCs increase phosphorylation of p38 MAPK in HL60 and K562 cells in a time-dependent manner. Synchronized tumor cells $\left(5 \times 10^{6}\right)$ were treated with UC-MSCs $\left(5 \times 10^{5}\right)$ for the indicated time. Protein extracts were then prepared and both phosphorylated and total p38 MAPK in the extracts were determined by Western blot analysis. Each lane contained $50 \mu \mathrm{g}$ protein. Actin was shown as a loading control. The results are representative of three independent experiments.

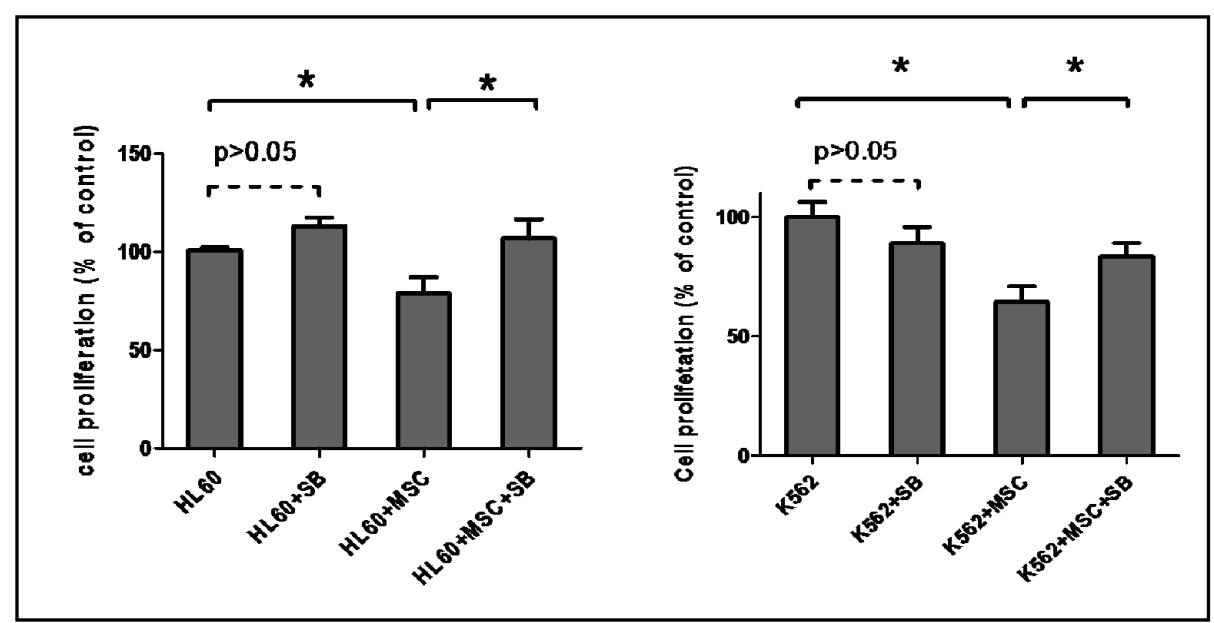

Fig. 3. Pharmacological inhibition of p38 MAPK partially abrogates MSC-induced proliferation suppression in HL60 and K562 cells. $10^{4}$ tumor cells were treated with $10^{3}$ UC-MSCs, $10 \mu \mathrm{M}$ of SB203580 (SB), or in combinations (SB $30 \mathrm{~min}$ before MSC) as indicated for $72 \mathrm{~h}$ and the cell proliferation was determined by BrdU incorporation assay. Values are expressed as percentage of untreated cells (\% of control) and each bar represents the mean $\pm \mathrm{SD}$ of triplicate determinations. ${ }^{*} \mathrm{P}<0.05$ versus tumor cells with MSCs treatment only. The results are representative of three independent experiments.

$48 \mathrm{~h}$ post-transfection, tumor cells were treated with UC-MSCs. Cell proliferation of tumor cells was assayed $72 \mathrm{~h}$ after treatment with UC-MSCs, and cell cycle progression was analyzed $48 \mathrm{~h}$ after coculture with UC-MSCs.

\section{Western blot analysis}

After indicated treatment, tumor cells were harvested and lysed in RIPA buffer (50 mM Tris-HCl, pH 7.6, $150 \mathrm{mM} \mathrm{NaCl}$, $1 \%$ NP40, 0.25\% deoxycholic acid, 1 mM EGTA, 1 mM EDTA, 1 $\mathrm{mM} \mathrm{NaF}, 1 \mathrm{mM} \mathrm{Na} \mathrm{VO} 4)$ with protease inhibitor cocktail (Sigma). Protein concentration of the lysates was quantified by the Bradford protein assay (Pierce Rockford, IL). Equal amounts of protein $(50 \mu \mathrm{g})$ were electrophoresed in $5 \%$ or $15 \%$ sodium dodecyl sulfate-polyacrylamide gel and transferred to polyvinyldifluoride membranes (Millipore, Bedford, MA). The membranes were blocked for $1 \mathrm{~h}$ with $5 \%$ bovine serum albumin (BSA) in Phosphate Buffered Saline (PBS), and incubated with primary antibodies diluted 1: 1000 in $5 \% \mathrm{BSA}$ at $4{ }^{\circ} \mathrm{C}$ overnight. After washing, membranes were incubated with the corresponding horseradish peroxidase-conjugated secondary antibody (Pierce) diluted 1: 4000 in 5\% BSA. The immunocomplexes were visualized with an enhanced chemiluminescence detection kit according to the manufacturer's instructions (Pierce). When necessary,

Tian/Yang/Ren/Han/Lu/Ma/Zhang/Han 


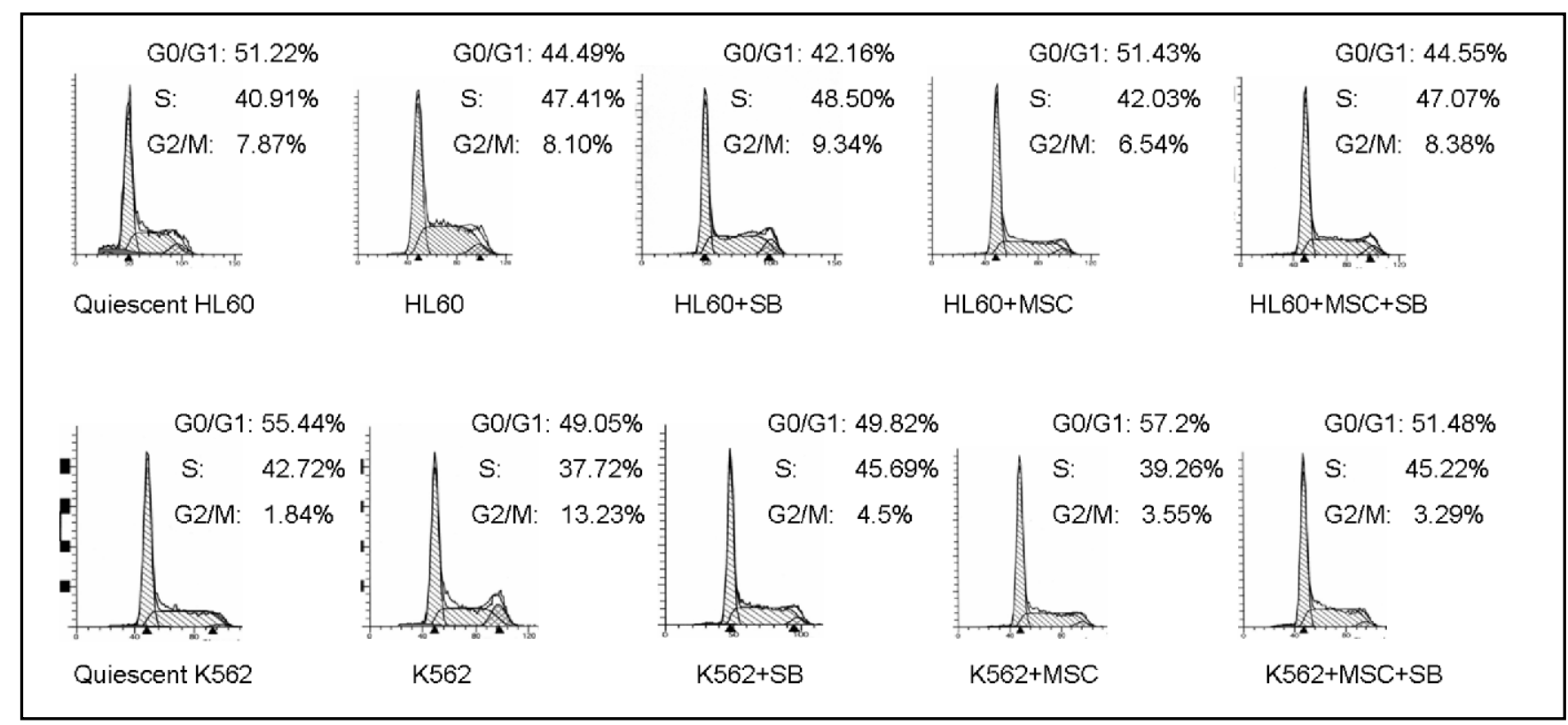

Fig. 4. Pharmacological inhibition of p38 MAPK partially reverses MSC-induced G0/G1 phase arrest in HL60 and K562 cells. Synchronized tumor cells $\left(10^{6}\right)$ were treated with $10^{5}$ UC-MSCs, $10 \mu \mathrm{M} \mathrm{SB} 203580$ (SB), or in combinations (SB 30 min before MSC) as indicated and cell-cycle progression was analyzed after $48 \mathrm{~h}$. Untreated tumor cells were kept as controls. Percentages of cells in each phase are shown in the upper corner of each cell-cycle graph. The results are representative of three independent experiments.

membranes were stripped by incubation in $62.5 \mathrm{mM}$ Tris- $\mathrm{HCl}$ (pH 6.8), 2\% SDS, and $100 \mathrm{mM} 2$-mercaptoethanol for $30 \mathrm{~min}$ at $50{ }^{\circ} \mathrm{C}$. After washing, membranes were reprobed with other antibodies.

\section{Statistical Analysis}

Data are presented as mean $\pm \mathrm{SD}$. One-way ANOVA analysis of variance was used to make comparisons of parameters among groups. P less than 0.05 was considered significant. The SPSS 11.5 software package was used for the statistical tests.

\section{Results}

UC-MSCs inhibit tumor-cell proliferation by G0/ G1 phase arrest in the absence of apoptosis

To determine whether UC-MSC inhibit growth of leukemic tumor cells, we examined its effect on proliferatin of HL60 and K562 cell lines. These cells were treated with grading doses of UC-MSCs for $72 \mathrm{~h}$. We demonstrated that UC-MSCs inhibited tumor cell growth in a dose-dependently manner, with the maximum effect observed at a ratio of 1:1 (MSCs: tumor cells) (Fig. 1A). In order to determine whether UC-MSCs inhibit cell proliferation by inducing cell apoptosis, we stained the cells maintained in 1\% FCS medium with Annexin V/PI

p38 MAPK in Tumor Suppression by UC-MSCs and found that treatment of UC-MSCs (1:10) for $48 \mathrm{~h}$ did not induce cell apoptosis (Fig. 1B). To further explore the mechanism by which UC-MSCs inhibited tumor cell growth, We analyzed the cell-cycle progression of HL60 and K562 cells treated with UC-MSCs (1:10) for $48 \mathrm{~h}$ by flow cytometry. The fraction of HL60 in the G0/G1 phase was $44 \%$ in control, whereas, was $51 \%$ in the presence of UC-MSCs. Similarly, the fraction of K562 cells in the $\mathrm{G} 0 / \mathrm{G} 1$ phase was greatly increased in response to MSCs treatment (Fig. 1C). These results indicate that UC-MSCs inhibit growth of tumor cells by $\mathrm{G} 0 / \mathrm{G} 1$ arrest, not apoptosis induction.

UC-MSCs increase phosphorylation of $p 38$ $M A P K$ in tumor cells

It has been demonstrated that BM-MSCs modified the phosphorylation pattern of the p38 pathways in B-lymphocytes, accompanied with the proliferation inhibition of B-lymphocytes [13]. We thus examined whether MSCs had an effect on phosphorylation of p38 MAPK in tumor cells. Synchronized tumor cells were treated with MSCs for indicated time and the level of total and phosphorylated p38 MAPK were analyzed by Western blotting using the antibodies against these kinases. As shown in Fig. 2, UC-MSCs induced the phosphorylation of p38 MAPK in a time-dependent 
A

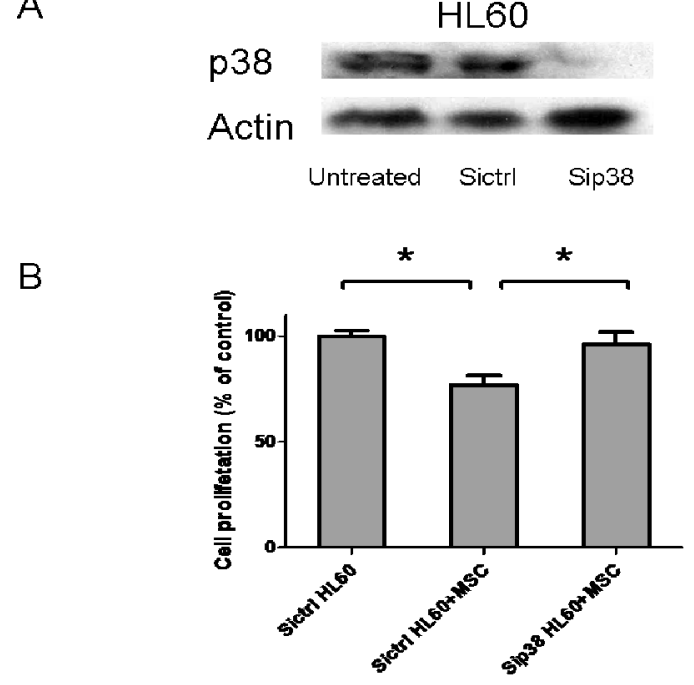

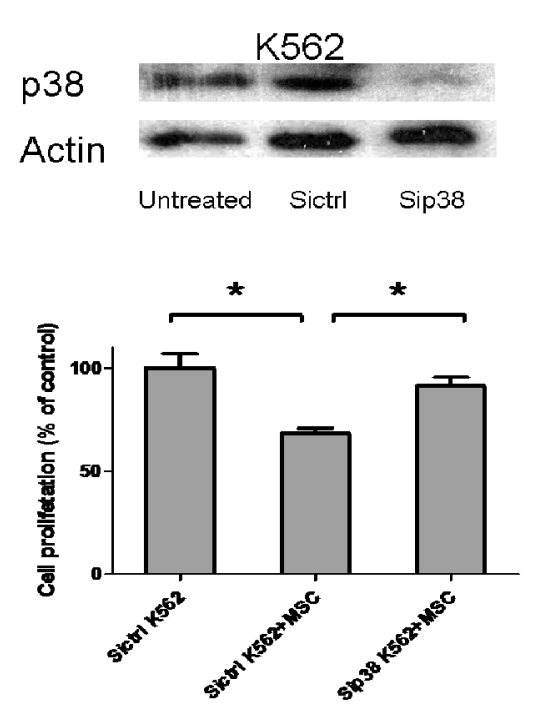

C

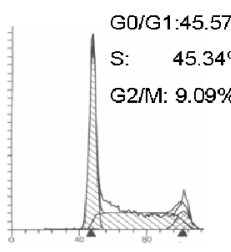

Sictrl HL6O
Sictrl HL60+MSC

Sip38 HL60+MSC
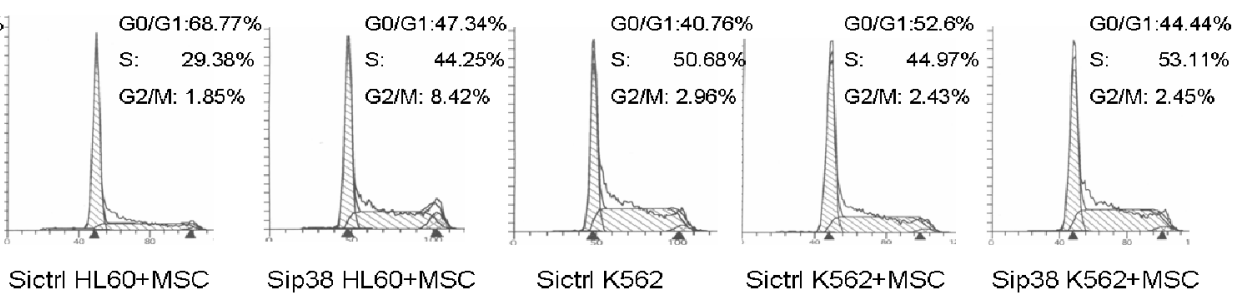

Fig. 5. siRNA down-regulation of p38 MAPK partially abrogates MSC-induced proliferation inhibition (B) and cell cycle arrest (C) in HL60 and K562 cells. (A) p38 MAPK expression is suppressed when the targeting siRNA was introduced. Tumor cells were transfected with negative control siRNA (siCtrl) or siRNA targeting p38 MAPK (sip38). Untreated tumor cells were kept as control. $72 \mathrm{~h}$ post-transfection, cells were harvested and lysed. Immunoblotting was performed using antibodies against p38MAPK. Actin was used as a loading control. The results are representative of three independent experiments. (B) Tumor cells were treated with siCtrl or sip38 for $48 \mathrm{~h}$ before harvested and quantified. Transfected tumor cells in 96 -well plates (10 ${ }^{4}$ per well) were cultured with or without UC-MSCs $\left(10^{3}\right.$ per well) for $72 \mathrm{~h}$ and then cell proliferation of tumor cells was determined by BrdU incorporation assay. Values are expressed as percentage of siCtrl cells without MSCs treatment (\% of control) and each bar represents the mean $\pm \mathrm{SD}$ of triplicate determinations. ${ }^{*} \mathrm{P}<0.05$ versus siCtrl cells with MSCs treatment. The results are representative of three independent experiments. (C) Tumor cells were treated with siCtrl or sip38 for $48 \mathrm{~h}$ before harvested and quantified. $10^{6}$ transfected tumor cells were cultured with or without $10^{5} \mathrm{UC}$-MSCs for $48 \mathrm{~h}$ and then the cell-cycle progression of tumor cells was analyzed by flow cytometry. Percentages of cells in each phase are shown in the upper corner of each cell-cycle graph. The results are representative of three independent experiments.

manner. The phosphorylation peaked at $20 \mathrm{~min}$ and declined thereafter.

Pharmacological inhibition of p 38 MAPK partially attenuates MSC-induced antiproliferation effect

To test whether p38 MAPK is critical for MSCinduced anti-proliferative effect, we treated HL60 and K562 cells with $10 \mu \mathrm{M}$ of SB203580 (a special inhibitor of p38 MAPK) for $30 \mathrm{~min}$ prior to the UC-MSCs treatment, then examined the growth of these cells. As shown in Fig. 3, the growth-inhibitory effect of UC-MSCs was distinctly blocked by the pretreatment with SB203580.

Pharmacological inhibition of p38 MAPK partially reverses the G0/G1 arrest of tumor cells mediated by UC-MSCS

To examine whether p38 MAPK activation is involved in MSC-induced cell cycle arrest of tumor cells, synchronized HL60 and K562 cells were preincubated with SB203580 $10 \mu \mathrm{M}$ for 30 min before treatment with

Tian/Yang/Ren/Han/Lu/Ma/Zhang/Han 


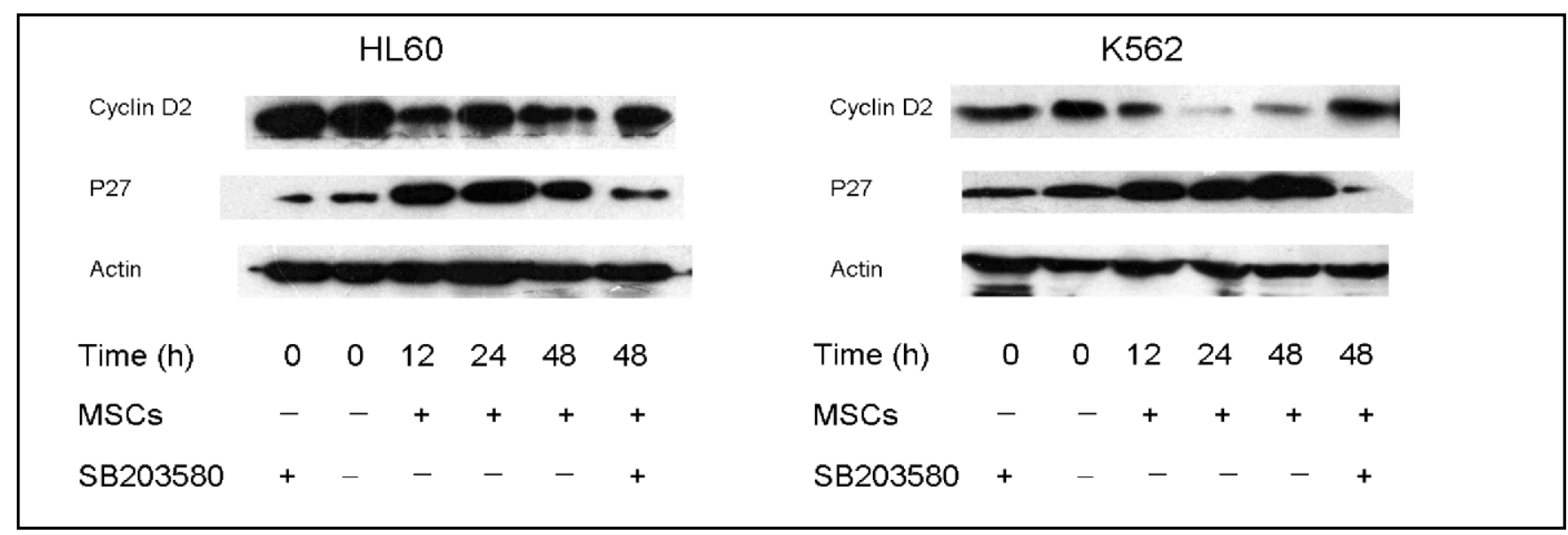

Fig. 6. UC-MSCs decrease the expression of cyclin D2 and increase the expression of $\mathrm{p} 27^{\mathrm{kip} 1}$ in tumor cells, while SB203580 reverses the effect. Tumor cells $\left(5 \times 10^{6}\right)$ were treated with UC-MSCs $\left(5 \times 10^{5}\right)$ for the indicated time, or pretreated with $10 \mu \mathrm{M}$ of SB203580 for 30 minutes and then exposed to UC-MSCs $\left(5 \times 10^{5}\right)$ for $48 \mathrm{~h}$. Protein extracts were then prepared and the expression of cyclin D2 and p27 $7^{\mathrm{kip} 1}$ in the extracts were determined by Western blot analysis. Each lane contained $50 \mu \mathrm{g}$ protein. Actin was shown as a loading control. The results are representative of three independent experiments.

UC-MSCs. Cell cycle analysis showed that G0/G1 phase arrest in HL60 and K562 cells mediated by UC-MSC was also partially reversed by the pretreatment with SB203580 (Fig. 4). The results suggest that activation of p38 MAPK by UC-MSCs is important for G0/G1 arrest of tumor cells.

Knockout of $p 38$ MAPK by siRNA partially reverses MSC-mediated growth inhibition of tumor cells

Pharmacological inhibitors are usually associated with nonspecific inhibitory effect, therefore we used specific p38 MAPK siRNA to knockout its expression. As shown in Fig. 5A, treatment of tumor cells with p38 siRNA (sip38) effectively reduced the expression of $\mathrm{p} 38$ MAPK as determined with specific antibody of $\mathrm{p} 38$. We then examined MSC-induced cell proliferation of tumor cells treated with siRNA. Similar to the effect of SB203580 (Fig. 3), knockout of p38 attenuated MSCinduced proliferation inhibition (Fig. 5B). We further analyzed the effects of p38 knockout on MSC-induced cell cycle arrest by flow cytometry. Fig. $5 \mathrm{C}$ shows that knockout of p38 significantly reversed the G0/G1 arrest caused by UC-MSCs. These results confirm that p38 MAPK is required for the growth inhibition of tumor cells mediated by UC-MSCs.

p38 MAPK in Tumor Suppression by UC-MSCs
UC-MSCs modulate the expression of cell cycle regulators through $\mathrm{p} 38 \mathrm{MAPK}$

To better understand the molecular mechanisms involved in MSC-induced growth inhibition of tumor cells, we investigated the expression level of cell cycle regulators which have been implicated in the growth inhibition of tumor cells caused by BM-MSCs [1]. As shown in Fig. 6, UC-MSCs markedly decreased the expression of cyclin D2 and increased the expression of $\mathrm{p} 27^{\mathrm{kip} 1}$ in a time-course manner. Notably, the p38 MAPK inhibitor, SB203580, potently reversed the effect of UC-MSCs. These data suggest that MSC-induced modulation of cell cycle regulators requires p38 MAPK activation.

\section{Discussion}

MSCs are known to inhibit tumor growth of hematopoitic and non-hematopoietic origin in vitro. Cellcontact, DKK-1 and WNT signaling pathway have been described as modulating tumor-cell proliferation $[1,3]$. Here, we demonstrate for the first time that UC-MSCs is able to suppression the proliferation of leukemic tumor cells in a dose-dependent manner in vitro and $\mathrm{p} 38$ MAPK contributes to the inhibitory effect. Although p38 MAPK 
was implicated to be a tumor suppressor [10], p38 MAPK has not been reported to mediate tumor-cell suppression by MSCs. Our hypothesis that $\mathrm{p} 38$ MAPK is involved in the growth-suppression of leukemic tumor cells by UCMSCs in vitro was supported by the following facts: (1) UC-MSCs were able to induce the phosphorylation of p38 in a time-dependent manner; (2) Pharmacological inhibition or genetic silencing of the p38 MAPK partially abrogated the growth inhibitory effect of UC-MSCs; (3) In addition, UC-MSCs modulated the expression of cell cycle regulator through p38 MAPK pathway.

It has been reported that BM-MSCs mediated growth inhibition of immune cells and tumor cells by cell cycle arrest without induction of apoptosis [14-17]. Our results indicate that UC-MSCs treatment led to proliferation inhibition and $\mathrm{G} 0 / \mathrm{G} 1$ arrest of leukemic tumor cells, similar to the effect of BM-MSCs.

The p38 MAPKs affect a variety of intracellular responses, with well-recognized roles in inflammation, cell-cycle regulation, cell death, development, differentiation, senescence and tumorigenesis [9]. The involvement of p38 MAPK in cell growth inhibition is associated with the induction of G0/G1 arrest in various cell types depending on the stimulating signal [18-21]. The tumour-suppressive activity of p38 MAPK can largely be attributed to its modulatory effects on $\mathrm{G} 1 / \mathrm{S}$ and $\mathrm{G} 2 / \mathrm{M}$ cell cycle checkpoint [22], negative regulation of proliferation and induction of apoptosis [23-25]. However, the role of p38 MAPK in tumor-suppression by MSCs remains unclear. The findings herein showed that UCMSCs activated the p38 MAPK pathway in leukemic tumor cells, similar to the previous observation that MSCs modified the activation pattern of p38 MAPK in B lymphocytes [13]. Furthermore, we demonstrated that the p38 MAPK pathway was required for the growth inhibition of leukemic tumor cells induced by UC-MSCs because pharmacological inhibition or genetic silencing of p38 MAPK dramatically reversed the effect of UCMSCs.

Accumulating evidence is defining a critical role of p38 MAPK in cell cycle checkpoint. Actually, p38 MAPK may regulate cell cycle progression through multiple mechanisms. One means by which $\mathrm{p} 38$ blocks cell cycle may be to inhibit the expression of cyclin D [26, 27]. Cyclin D proteins are positive cell cycle regulators. They bind to and active cyclin dependent kinase 4 and $6(\mathrm{cdk} 4 /$ 6) and this active complex is essential for the transition to $\mathrm{S}$ phase and further cell proliferation. Unlike other cyclins that are periodically induced during the cell cycle progression, the levels of cyclin $\mathrm{D}$ are regulated by the extracellular mitogenic environment. Thus, cyclin D proteins are believed to serve as "links" between the extracellular environment and the core cell cycle apparatus [28]. p38 MAPK has been shown to reduce the levels of cyclin D by transcription downregulation [29] and proteosomal degradation [30]. Moreover it has been reported that MSC dramatically downregulated the expression of cyclin D2 in tumor cells [1]. Therefore the association between cyclin D2 and p38 MAPK in the growth suppression of tumor cells by UC-MSCs was dertermined in this study. We observed that the p38 MAPK chemical inhibitor, SB203580, partially reversed the downregulation of cyclin D2 mediated by UC-MSCs, suggesting that UC-MSCs decrease the expression of cyclin D2 through p38 pathway. However, the exact level at which cyclin D2 is regulated, and the potential components that p38 phosphorylates to decrease cyclin D2 expression remain unclear, which will require further investigation.

Another important mechanism by which p38 MAPK blocks cell cycle is to upregulate the expression of CDK inhibitor $\mathrm{p} 27^{\mathrm{kip} 1}$. It has been reported that $\mathrm{p} 38$ is involved in G1 cell-cycle arrest of iTregs controlled by cdk inhbitor p2 $7^{\mathrm{kip} 1}$ [31]. Sustained expression of $\mathrm{p} 27^{\mathrm{kip} 1}$ which coincidences with activation of $\mathrm{p} 38$ prevents phosphorylation of retinoblastoma protein $(\mathrm{Rb})$ by cdk 4 or 6. Hypophosphorylation of $\mathrm{Rb}$ allows for binding of the transcription factor E2F. Hence E2F-dependent geneexpression, which are necessary for entry into S-phase, is prevented.

Therefore we investigated the effect of UC-MSCs on $\mathrm{p} 27^{\mathrm{kipl}}$ expression in tumor cells. We observed that UC-MSCs treatment led to a marked increase of $\mathrm{p} 27^{\mathrm{kip} 1}$ expression in tumor cells. Notably, SB203580, significantly abrogated the upregulation of $\mathrm{p} 27^{\mathrm{kip} 1}$, suggesting that the p38 MAPK pathway is implicated in MSCs-induced growth inhibition, possibly through the upregulatin of p2 $7^{\mathrm{kip} 1}$.

The p38 MAPK is known to be activated by proinflammatory and stressful stimuli, while MSCs could secrete several pro-inflammatory cytokines, such as hepatocyte growth factor (HGF). It has been reported that treatment with HGF inhibits the proliferation of a number of tumor cell lines [32,33], and p38 is required for HGF's inhibitory effect on MSC proliferation [34]. Further studies are needed to identify the cytokines responsible for tumor suppression by MSCs and HGF may be one of the candidates.

Tian/Yang/Ren/Han/Lu/Ma/Zhang/Han 
In conclusion, our results provide new insight into how UC-MSCs modulate leukemic tumor growth in vitro. Although it is known that p38 MAPK is a suppressor of tumor development, this is the first report demonstrating that the p38 MAPK is required for leukemic tumor suppression by UC-MSCs. Our data bring new insights into clinical therapeutic trials using UC-MSC infusion for tumor-suppression purposes.

\section{Acknowledgements}

This study was supported by 863 projects from Ministry Science \& Technology of China (2006AA02A110), National Natural Science Foundation of China (30570357, 30600238 and 30900557) and Tianjin Municipal Science and Technology Commission (06YFSZSF01300 and 07JCYBJC11200). Disclosure: The authors declare no competing financial interests.

\section{References}

Ramasamy R, Lam EW, Soeiro I, Tisato 6 V, Bonnet D, Dazzi F: Mesenchymal stem cells inhibit proliferation and apoptosis of tumor cells: impact on in vitro tumor growth. Leukemia 2007;21:304-310.

Khakoo AY, Pati S, Anderson SA, Reid W, Elshal MF, Rovira, II, Nguyen AT, Malide D, Combs CA, Hall G, Zhang J, Raffeld M, Rogers TB, Stetler-Stevenson W, Frank JA, Reitz M, Finkel T: Human mesenchymal stem cells exert potent antitumorigenic effects in a model of Kaposi's sarcoma. J Exp Med 2006;203:1235-1247.

-3 Zhu Y, Sun Z, Han Q, Liao L, Wang J, Bian C, Li J, Yan X, Liu Y, Shao C, Zhao RC: Human mesenchymal stem cells inhibit cancer cell proliferation by secreting DKK-1. Leukemia 2009;23:925-933.

4 Ono K, Han J: The p38 signal transduction pathway: activation and function. Cell Signal 2000;12:1-13.

$>5$ Engel FB, Schebesta M, Duong MT, Lu $>_{10}$ G, Ren S, Madwed JB, Jiang H, Wang Y, Keating MT: p38 MAP kinase inhibition enables proliferation of adult mammalian 11 cardiomyocytes. Genes Dev 2005;19:1175-1187.
Saika S, Okada Y, Miyamoto T, Yamanaka O, Ohnishi Y, Ooshima A, Liu CY, Weng D, Kao WW: Role of p38 MAP kinase in regulation of cell migration and proliferation in healing corneal epithelium. Invest Ophthalmol Vis Sci 2004;45:100-109.

Pang $\mathrm{PH}$, Lin $\mathrm{YH}$, Lee $\mathrm{YH}$, Hou $\mathrm{HH}$, Hsu SP, Juan SH: Molecular mechanisms of p21 and p27 induction by 3methylcholanthrene, an arylhydrocarbon receptor agonist, involved in antiproliferation of human umbilical vascular endothelial cells. J Cell Physiol 2008;215:161-171.

Thornton TM, Rincon M: Non-classical p38 map kinase functions: cell cycle checkpoints and survival. Int $\mathbf{J}$ Biol Sci 2009;5:44-51.

Hui L, Bakiri L, Stepniak E, Wagner EF: p38alpha: a suppressor of cell proliferation and tumorigenesis. Cell Cycle 2007;6:2429-2433.

Han J, Sun P: The pathways to tumor suppression via route $\mathrm{p} 38$. Trends Biochem Sci 2007;32:364-371.

Vaidya AA, Sharma MB, Kale VP: Suppression of p38-stress kinase sensitizes quiescent leukemic cells to antimitotic drugs by inducing proliferative responses in them. Cancer Biol Ther 2008;7:1232-1240.
Lu LL, Liu YJ, Yang SG, Zhao QJ, Wang X, Gong W, Han ZB, Xu ZS, Lu YX, Liu D, Chen ZZ, Han ZC: Isolation and characterization of human umbilical cord mesenchymal stem cells with hematopoiesis-supportive function and other potentials. Haematologica 2006;91:1017-1026.

Tabera S, Perez-Simon JA, Diez-Campelo M, Sanchez-Abarca LI, Blanco B, Lopez A, Benito A, Ocio E, Sanchez-Guijo FM, Canizo C, San Miguel JF: The effect of mesenchymal stem cells on the viability, proliferation and differentiation of $\mathrm{B}$ lymphocytes. Haematologica 2008;93:1301-1309.

Corcione A, Benvenuto F, Ferretti E, Giunti D, Cappiello V, Cazzanti F, Risso M, Gualandi F, Mancardi GL, Pistoia V, Uccelli A: Human mesenchymal stem cells modulate B-cell functions. Blood 2006;107:367-372.

5 Kim JA, Hong S, Lee B, Hong JW, Kwak JY, Cho S, Kim CC: The inhibition of Tcells proliferation by mouse mesenchymal stem cells through the induction of p16INK4A-cyclin D1/cdk4 and p21waf1, p27kip1-cyclin E/cdk2 pathways. Cell Immunol 2007;245:1623. 
16 Lu YR, Yuan Y, Wang XJ, Wei LL, Chen YN, Cong C, Li SF, Long D, Tan WD, Mao YQ, Zhang J, Li YP, Cheng JQ: The growth inhibitory effect of mesenchymal stem cells on tumor cells in vitro and in vitro. Cancer Biol Ther 2008;7:245-251.

17 Ramasamy R, Fazekasova H, Lam EW, Soeiro I, Lombardi G, Dazzi F: Mesenchymal stem cells inhibit dendritic cell differentiation and function by preventing entry into the cell cycle. Transplantation 2007;83:71-76.

18 Jeon HK, Choi SU, Jung NP: Association of the ERK $1 / 2$ and p38 kinase pathways with nitric oxide-induced apoptosis and cell cycle arrest in colon cancer cells. Cell Biol Toxicol 2005;21:115-125.

19 Moon SK, Jung SY, Choi YH, Lee YC, Patterson C, Kim CH: PDTC, metal chelating compound, induces $\mathrm{G} 1$ phase cell cycle arrest in vascular smooth muscle cells through inducing $\mathrm{p} 21 \mathrm{Cip} 1$ expression: involvement of p38 mitogen activated protein kinase. J Cell Physio 2004;198:310-323.

20 Seay U, Sedding D, Krick S, Hecker M, Seeger W, Eickelberg O: Transforming growth factor-beta-dependent growth inhibition in primary vascular smooth muscle cells is p38-dependent. J Pharmacol Exp Ther 2005;315:10051012.

-21 Yao YQ, Ding X, Jia YC, Huang CX, Wang YZ, Xu YH: Anti-tumor effect of beta-elemene in glioblastoma cells depends on p38 MAPK activation. Cancer Lett 2008;264:127-134.
22 Ambrosino C, Nebreda AR: Cell cycle regulation by $\mathrm{p} 38$ MAP kinases. Biol Cell 2001;93:47-51.

23 Bulavin DV, Fornace AJ Jr: p38 MAP kinase's emerging role as a tumor suppressor. Adv Cancer Res 2004;92:95118 .

$>24$ Kummer JL, Rao PK, Heidenreich KA: Apoptosis induced by withdrawal of trophic factors is mediated by $\mathrm{p} 38$ mitogen-activated protein kinase. J Biol Chem 1997;272:20490-20494.

25 She QB, Bode AM, Ma WY, Chen NY, Dong Z: Resveratrol-induced activation of p53 and apoptosis is mediated by extracellular-signal-regulated protein kinases and p38 kinase. Cancer Res 2001;61:1604-1610.

26 Cha H, Wang X, Li H, Fornace AJ Jr.: A functional role for p38 MAPK in modulating mitotic transit in the absence of stress. J Biol Chem 2007;282:2298422992.

27 Kida A, Kakihana K, Kotani S, Kurosu T, Miura O: Glycogen synthase kinase-3beta and p38 phosphorylate cyclin D2 on Thr280 to trigger its ubiquitin/ proteasome-dependent degradation in hematopoietic cells. Oncogene 2007;26:6630-6640.

Sherr CJ, Roberts JM: Living with or without cyclins and cyclin-dependent kinases. Genes Dev 2004;18:2699-2711.
Lavoie JN, L'Allemain G, Brunet A, Muller R, Pouyssegur J: Cyclin D1 expression is regulated positively by the p42/p44MAPK and negatively by the p38/HOGMAPK pathway. J Biol Chem 1996;271:20608-20616.

Casanovas O, Miro F, Estanyol JM, Itarte E, Agell N, Bachs O: Osmotic stress regulates the stability of cyclin D1 in a p38SAPK2-dependent manner. J Biol Chem 2000;275:35091-35097.

Adler HS, Kubsch S, Graulich E, Ludwig S, Knop J, Steinbrink K: Activation of MAP kinase p38 is critical for the cellcycle-controlled suppressor function of regulatory T cells. Blood 2007;109:43514359.

Shiota G, Rhoads DB, Wang TC, Nakamura T, Schmidt EV: Hepatocyte growth factor inhibits growth of hepatocellular carcinoma cells. Proc Natl Acad Sci U S A 1992;89:373-377.

Tajima H, Matsumoto K, Nakamura T: Hepatocyte growth factor has potent anti-proliferative activity in various tumor cell lines. FEBS Lett 1991;291:229-232.

-34 Forte G, Minieri M, Cossa P, Antenucci D, Sala M, Gnocchi V, Fiaccavento R, Carotenuto F, De Vito P, Baldini PM, Prat M, Di Nardo P: Hepatocyte growth factor effects on mesenchymal stem cells: proliferation, migration, and differentiation. Stem Cells 2006;24:2333. 